Rev. Saúde públ., S. Paulo, 24(4): 253-8, 1990

\title{
ASPECTOS SÓCIO-ECONÔMICOS DOS INDIVÍDUOS COM MALÁRIA IMPORTADA NA REGIÃO METROPOLITANA DE SÃO PAULO, BRASIL. I - CARACTERIZAÇÃO DA POPULAÇÃO E CONHECIMENTO SOBRE A DOENÇA*
}

\author{
Maria José Chinelatto Pinheiro Alves* \\ Luiz Carlos Barradas Barata** \\ Rita de Cássia Barradas Barata*** \\ Maria do Carmo R. Rodrigues de Almeida** \\ Eliana B. Gutierrez** \\ Dalva Marli Valério Wanderley** \\ José Carlos Rehder de Andrade ${ }^{* *}$
}

\begin{abstract}
ALVES, M. J. C. P. et al. Aspectos sócio-econômicos dos indivíduos com malária importada ná região metropolitana de São Paulo, Brasil. I - Caracterização da populaçăo e conhecimento sobre a doença. Rev. Saúde publ., S.Paulo, 24: 253-8, 1990.
\end{abstract}

RESUMO: Com o objetivo de conhecer o perfil sócio-econômico dos indivíduos que se deslocaram das áreas endêmicas de malária do país, foram estudadas 566 pessoas com suspeita de malária que procuraram a confirmação diagnóstica no Laboratório de Malária da Região Metropolitana de São Paulo da Superintendência de Controle de Endemias (SUCEN). As informações foram obtidas através da aplicação de formulário, no período de novembro de 1986 a junho de 1987 . Da população estudada, $345(61,0 \%)$ residiam na área endêmica, $479(84,6 \%)$ eram do sexo masculino, $513(90,7 \%)$ estavam na faixa etária de 15 a 55 anos e $307(54,2 \%)$ apresentaram hemoscopia positiva para plasmódio. Com relação à ocupacão na área de transmissão, observou-se que 109 (19,3\%) estavam ligados a atividade de extração de minerais, $74(13,2 \%)$ à agricultura e $46(8,1 \%)$ à atividade de transporte. A análise da escolaridade mostrou que 486 $(85,9 \%)$ tinham $1^{\circ}$ ou $2^{\circ}$ grau. Quanto do conhecimento sobre a doença, $384(67,8 \%)$ declararam pelo menos 1 malária anterior e $491(86,8 \%)$ associavam à doença a presença do vetor. Dentre os 221 indivíduos residentes em São Paulo, 207 (93,7\%) conheciam o risco de contrair malária por ocasião do deslocamento para área de transmissão. Daqueles residentes na área endêmica, $336(97,4 \%)$ tinham conhecimento do risco de contrair a doença naquela regiăo. $O$ intervalo transcorrido entre os primeiros sintomas e a procura de atendimento médico em $386(68,2 \%)$ individuos variou de 0 a 3 dias. As freqüências das variáveis estudadas mostraram de acordo com o resultado hemoscópico e o local da residência, diferenças estatísticas relevantes. prática.

DESCRITORES: Malária, epidemiologia. Fatores sócio-econômicos. Conhecimentos, atitudes e

\section{INTRODUÇÃO}

No Brasil, a incidência de malária vem aumentando progressivamente a partir de 1970, ano em que foi registrado o menor número de casos $(52.469)^{13}$. Informações da Superintendência de Campanhas de Saúde Pública (SUCAM) o registro de 508.864 lâminas positivas em 1987, em sua grande maioria restritas à Região Amazônica.

Nessa citada região, a política de expansão foi intensificada no início da década de setenta, quando a ocupação de terras e o aproveitamento dos re- cursos naturais passaram a atrair representativo contingente populacional. O crescimento demográfico verificado na Amazônia foi acompanhado de aumento da transmissão de doenças infecciosas, particuiarmente a malária ${ }^{13}$.

As modificações ocorridas nas áreas de atração, pelos deslocamentos da população, contribuíram para a disseminação da endemia na Região Amazônica e o seu reflexo para outros Estados da Federação, onde a transmissão da doença encontra-se controlada ${ }^{7,8}$. Deste modo, a estrutura social foi alterada tanto nas áreas de evasão como nas de recepção de migrantes, conduzindo a mudança nos

\footnotetext{
* Realizado com apoio financeiro do Conselho Nacional de Desenvolvimento Científico e Tecnológico-Programa Integrado de Doenças Endêmicas (CNPq-PIDE). Processo n² 40.0911/85.

* * Superintendência de Controle de Endemias - Rua Paula Souza, 166 - 01027 - São Paulo, SP - Brasil.

* * Faculdade de Ciências Médicas da Santa Casa de São Paulo - Rua Dr. Cesário Mota Jr., 112 — 01221 - São Paulo, SP $-\mathrm{B}$ rasil.
} 
perfis epidemiológicos de algumas classes sociais, refletindo nos riscos individuais de doença ${ }^{3}$.

No Estado de São Paulo, a situação da endemia que era considerada estável até o ano de 1982, quando se registrava uma média de 1.200 casos ao ano, vem sofrendo modificações avaliadas a partir do aumento da incidência. Em 1987, foram notificados 3.066 casos, dos quais cerca de $90 \%$ eram importados e originários da Região Amazônica, principalmente dos Estados de Rondônia, Mato Grosso e Pará ${ }^{12}$.

De acordo com Aguedelo ${ }^{1}$, embora se reconheça o caráter social da malária, ainda é pouco explorado o conhecimento deste aspecto da doença, que não tem sido incorporado tanto nas interpretações como nas práticas de intervenção. Projetos de investigação são ainda incipientes em nosso meio, em conseqüência das dificuldades metodológicas na construção de modelos para a execução de trabalhos de campo, que incluam os processos biológicos com os econômicos e sociais.

Atendendo às necessidades do Programa de Controle executado pela Superintendência de Controle de Endemias (SUCEN) da Secretaria de Estado da Saúde, em 1984, foi proposto o estudo de alguns fatores envolvidos na transmissão da malária. Assim, com o objetivo de conhecer o perfil sócio-económico e os conceitos sobre a doença dos indivíduos que se deslocaram da área endêmica de malária do país e procuraram o Laboratório de Malária da SUCEN, para diagnóstico e tratamento, foi desenvolvido o presente estudo. Procurou-se estabelecer as possíveis diferenças entre aqueles que apresentaram resultado hemoscópico positivo e resultado negativo e com residência declarada na área endêmica e em São Paulo.

Os objetivos específicos da pesquisa foram: caracterizar a đistribuição por sexo e faixa etária; conhecer a situação de trabalho, o setor e ramo de atividade e a ocupação do indivíduo; determinar o grau de instrução e classificar os níveis de renda; avaliar o nível de conhecimento e compreensão da malária como agravo à saúde; identificar as condicōes de moradia no local provável da infecção; estudar as razões do deslocamento para a área endêmica, e desta para o Município de São Paulo, classificar os deslocamentos em movimentos circulatórios ou migratórios; estimar a oferta de recursos de assistência em saúde utilizados e à disposição.

Neste artigo, são apresentados os dados da identificação do indivíduo e conhecimento sobre a doença, através do estudo das seguintes variáveis: residência, sexo, idade, ocupação, instrução, conhecimento e compreensão da malária.

\section{MATERIAL E METODO}

O presente estudo foi realizado na Região Me- tropolitana de São Paulo (RMSP), o maior centro urbano e industrial do Brasil, com uma população de aproximadamente 16 milhões (13,3\% da população do país) ${ }^{5}$. Este processo de crescimento teve início a partir da década de 30 , representando este centro, hoje, pólo de atração de migrantes do próprio Estado de São Paulo e de outros Estados à procura de trabalho e melhores condições de vida. Esta região tem sido responsável pelo diagnóstico de $40 \%$ dos casos notificados de malária no Estado de São Paulo ${ }^{12,14}$.

O universo de estudo foi constituído por 566 indivíduos procedentes de região endêmica do país e que procuraram, para diagnóstico e tratamento, segundo procedimentos anteriormente descritos ${ }^{14,15}, 0$ Laboratório de Malária do Serviço Regional 1 da SUCEN, situado na cidade de São Paulo, no período de novembro de 1986 a junho de 1987.

Foi aplicado a todos os indivíduos, antes do resultado hemoscópico, para o qual foi utilizada a técnica da gota espessa, um formulário contendo 78 questōes. Estas permitiram caracterizar os indivíduos quanto à residência, sexo, faixa etária, ocupação, escolaridade e conhecimento da malária. Em relação à variável ocupação foi considerada a atividade declarada no momento em que o indivíduo supostamente contraiu a doença. Quanto à escolaridade, foram considerados analfabetos aqueles que não sabiam ler, apenas alfabetizados aqueles que sabiam ler, porém não haviam freqüentado nenhuma instituição de ensino; de nível médio os que freqüentavam o primeiro ou o segundo grau de forma completa ou incompleta, o mesmo ocorrendo com o nível superior.

Para avaliar o grau de conhecimento sobre a doença, foram utilizadas as seguintes variáveis: número de vezes que contraiu a malária, número de ocasiōes em que procurou a SUCEN e noçôes sobre o mecanismo de transmissão da doença.

$\mathrm{Na}$ interpretação de alguns dados foram utilizados testes não paramétricos de significância (qui-quadrado).

\section{RESULTADOS E DISCUSSÃO}

A distribuição da população estudada, segundo local de residência, indicou que $345(61,0 \%)$ moravam na área endêmica e $221(39,0 \%)$ referiram residir na Região Metropolitana de São Paulo. Quanto ao sexo (Tabela 1), $479(84,6 \%)$ são do sexo masculino, corroborando as observações de estudos anteriores que revelaram que em média $85 \%$ dos diagnósticos efetuados no Estado de São Paulo referiram-se a indivíduos do sexo masculino, em idade produtiva14,15. Esta participação maior reflete, provavelmente, o deslocamento mais intenso de homens para áreas de maior risco de transmissão para desenvolver atividades nos garimpos, projetos de colonização recente e atividades de 
TABELA 1

Distribuiçăo de indivíduos segundo sexo e resultado hemoscópico. Laboratório de Malária da Região Metropolitana de São Paulo, novembro de 1986 a junho de 1987

\begin{tabular}{lrrrrrr}
\hline \multirow{2}{*}{ Sexo } & \multicolumn{3}{c}{ Hemoscopia } & \multicolumn{2}{c}{ Total } \\
& \multicolumn{2}{c}{ Positiva } & \multicolumn{2}{c}{ Negativa } & & \\
& $\mathbf{N}^{\mathbf{2}}$ & $\%$ & $\mathbf{N}^{\mathbf{2}}$ & $\%$ & N & $\%$ \\
\hline Masculino & 271 & 56,6 & 208 & 43,4 & 479 & 84,6 \\
Feminino & 36 & 41,1 & 51 & 58,6 & 87 & 15,4 \\
\hline Total & 307 & & 259 & & 566 & 100,0 \\
$\%$ & 54,2 & & 45,8 & & 100,0 & \\
\hline
\end{tabular}

TABELA 2

Distribuiçăo dos indivíduos segundo a ocupação. Laboratório de Malária da Região Metropolitana de São Paulo, novembro de 1986 a junho de 1987

\begin{tabular}{lrr} 
Ocupação exercida na área de transmissão & N. ${ }^{\circ}$ & $\%$ \\
\hline $\begin{array}{l}\text { Empregado em atividade extrativa e na } \\
\text { agricultura }\end{array}$ & 183 & 32,4 \\
Empregado na prestação de serviço e no & 155 & 27,4 \\
$\quad$ comércio & & \\
Empregado em atividades de transporte, & & \\
$\quad$ construção civil e na indústria de & 91 & 16,0 \\
$\quad$ transformação & 17 & 3,0 \\
Profissionais liberais & 23 & 4,0 \\
Desempregados & 11 & 2,0 \\
Outras atividades & 81 & 14,3 \\
Atividades não declaradas & 5 & 0,9 \\
Sem atividades (menor e estudante) & 566 & 100,0 \\
\hline Total & &
\end{tabular}

transporte.

No tocante aos resultados da hemoscopia (Tabela 1), observa-se diferença $(p<0,05)$ entre mulheres com diagnóstico negativo em relação aos homens. Esta diferença, traduzida por um percentual maior de mulheres com diagnóstico negativo, pode ser explicada pelo fato de a mulher recorrer a serviços médicos com mais freqüência do que o homem, muitas vezes com sintomatologia pouco característica, diminuindo a chance de confirmação diagnóstica. Este aspecto foi bem caracterizado por Barros ${ }^{2}$ (1983) e Carvalho e col.4 (1988) em estudos de morbidade e utilização de serviços de saúde.

A análise das faixas etárias (Fig.) mostra uma concentração de indivíduos a partir dos 20 anos, não apresentando diferença na distribuição entre as populaçðes com hemoscopia positiva e negativa.

Relacionando os dados constantes da Tabela 1 e da Figura, verifica-se ser de pequena importância o deslocamento de agrupamentos familiares da re- gião endêmica, o que é indicado pela pequena participação de crianças e mulheres na população estudada, aspecto epidemiológico da malária nesse Estado, já observado em estudos anteriores ${ }^{15,16}$.

A distribuição dos indivíduos de acordo com a ocupação exercida no local de transmissão (Tabela 2) indica que, entre os 566 pesquisados, houve maior concentraçăo $(183-32,4 \%)$ daqueles inseridos nas atividades de extração mineral e agricultura, representando, respectivamente, 109 (19,2\%) e 74(13,2\%). Embora já se tivesse conhecimento de maior participação nessas atividades ${ }^{15}$, um fato novo é a frequêência $(27,4 \%)$ de portadores de malária envolvidos na prestação de serviços e comércio encontrada na presente pesquisa. Nas 91 respostas obtidas quanto às atividades de construção civil, indústria de transformação e transporte, observa-se que o percentual encontrado nesta última $8,1 \%$ (46) difere daquele referido no estudo citado anteriormente, quando o motorista de transporte contribui com $15,4 \%$. Salienta-se que no referido artigo ${ }^{15}$ foram analisados todos os casos de malária notificados no Estado de São Paulo, em 1985.

Observa-se que $81(14,3 \%)$ indivíduos não declararam a ocupação exercida na área de transmissão. Este percentual de "não respostas" indica que alguns pontos não foram devidamente observados no desenrolar da pesquisa, tanto em nível da aplicação dos formulários como na supervisão dos mesmos. Segundo Iversson ${ }^{6}$, isto pode ser em decorrência de barreira de comunicação entre entrevistador e entrevistado.

Quanto à escolaridade (Tabela 3), nota-se grande concentração de indivíduos com nível médio, em decorrencia de como foram agrupadas as categorias para análise, uma vez que todos aqueles que freqüentaram o primeiro ou segundo grau, de forma completa ou incompleta, foram classificados neste nível.

\section{TABELA 3}

Distribuição dos indivíduos segundo a escolaridade. Laboratório de Malária da Região Metropolitana de São Paulo, novembro de 1986 a junho de 1987

\begin{tabular}{lrr}
\hline Escolaridade & $\mathrm{N}$ e & $\%$ \\
\hline Analfabeto & 9 & 1,6 \\
Alfabetizado & 19 & 3,3 \\
Nível médio & 486 & 85,9 \\
Nível superior & 50 & 8,9 \\
Não sabe/Sem respostas & 2 & 0,3 \\
\hline Total & 566 & 100,0 \\
\hline
\end{tabular}

Com relação ao conhecimento sobre a doença, verifica-se que $93,7 \%$ dos indivíduos residentes em São Paulo que se deslocaram das áreas de trans- 


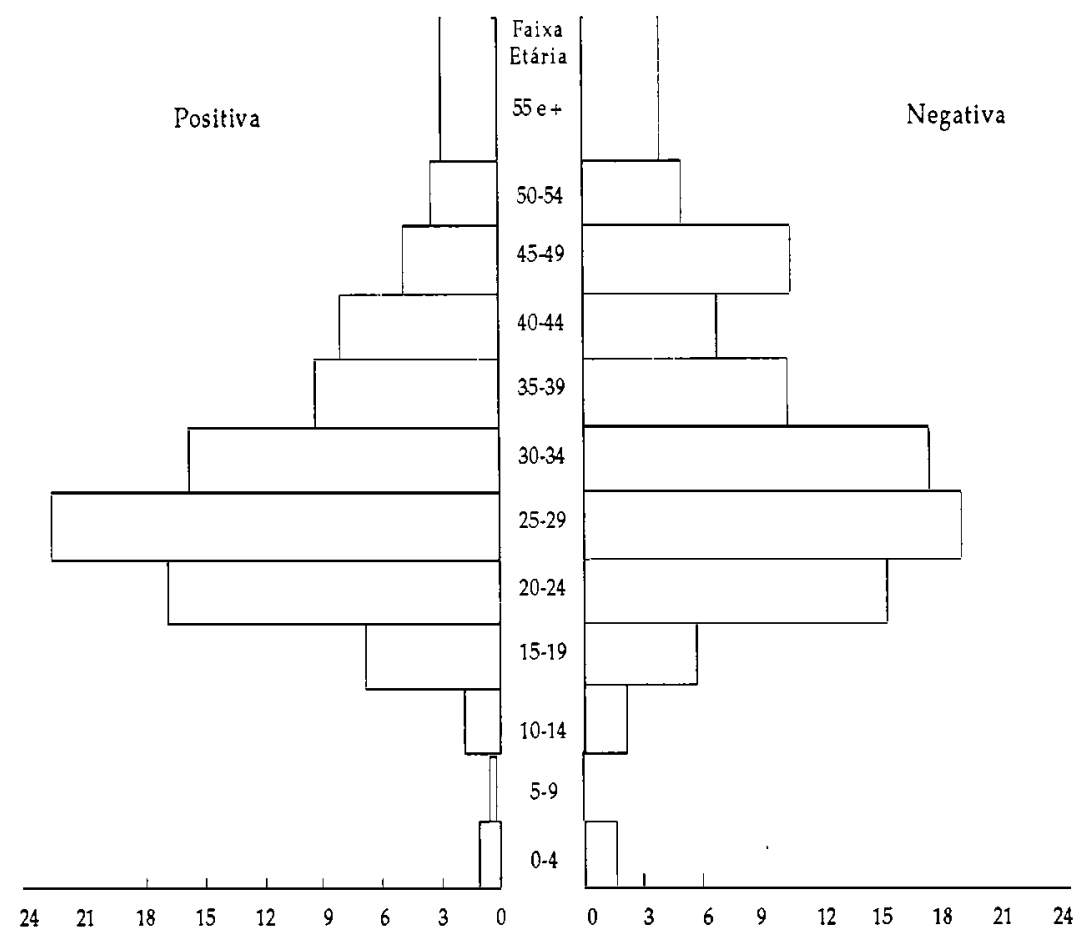

Figura 1 - Distribuição etária dos indivíduos segundo o resultado da hemoscopia.

Laboratório de Malária da Região Metropolitana de São Paulo, novembro de 1986 a junho de 1987.

missão conheciam o risco de contrair malária. Quando analisada a população total (566 indivíduos) com hemoscopia positiva e negativa, relacionando com o passado malárico, $384(69,1 \%)$ referiam malária anterior. Entre esses pacientes não foi observada diferença significativa entre o número médio de malárias anteriores. Salienta-se que $126(32,9 \%)$ doentes referiam mais de 10 malárias anteriores. Este dado sugere que esta categoria não foi devidamente explorada, não permitindo a distribuição dos mesmos de forma a possibilitar melhor análise.

Os resultados encontrados quanto ao conhecimento do modo de transmissão indicam que $86,8 \%$ dos inđivíduos associam a doença com a presença do vetor, podendo indicar conhecimento anterior da doença, bem como contato e permanència dos indivíduos em áreas endêmicas. Em pesquisa realizada no Projeto Machadinho, Estado de Rondônia, Sawyer e col. ${ }^{11}$ encontraram situação semelhante, muito embora o conhecimento sobre o mecanismo de transmissão da doença não seja completo.

Entre a população estudada, 531 individuos
(93,9\%) declararam não possuir hábito de ingerir medicamentos para não ter malária e $35(6,1 \%)$ informaram utilizar drogas preventivas. Os medicamentos mais citados foram: cloroquina, amodiaquina, sulfadoxina, pirimetamina, quinino, primaquina. No entanto, a questão poderia ter sido formulada de forma mais específica, uma vez que ..."não ter malária..." pode ser entendido tanto como prevenção de um ataque malárico ou como abortamento de uma crise inicial.

Com referência à distribuição dos indivíduos quanto à utilização de "receitas caseiras para não pegar malária" (Tabela 4), observa-se que, entre as 676 respostas obtidas dos 566 indivíduos entrevistados, $392(58,0 \%)$ não fazem uso de nenhum preventivo para não ter a doença. Foi verificada diferença significativa ( $p<0,025$ ) entre as respostas dos indivíduos com hemoscopia positiva e negativa. É provável que aqueles com diagnóstico positivo tivessem registro de episódios maláricos anteriores, possuindo conhecimento e prática da utilização de plantas.

Entre as plantas utilizadas, foram citadas: pa- 


\section{TABELA 4}

Distribuição de indivíduos segundo "receitas caseiras" utilizadas para prevenir malária e resultado de hemoscopia. Laboratório de Malária da Região Metropolitana de São Paulo, novembro de 1986 a junho de 1987

\begin{tabular}{|c|c|c|c|}
\hline \multirow{3}{*}{$\begin{array}{l}\text { Receitas } \\
\text { caseiras }\end{array}$} & \multicolumn{3}{|c|}{ Hemoscopia } \\
\hline & Positiva & Negativa & Total \\
\hline & $\mathrm{N}^{\circ} \quad \%$ & $\mathrm{~N}^{\circ} \quad \%$ & $\mathrm{~N}^{\mathrm{e}}$ \\
\hline
\end{tabular}

\begin{tabular}{|c|c|c|c|c|c|c|}
\hline $\begin{array}{l}\text { Alho ou } \\
\text { chá de alho } \\
\text { Chá de boldo } \\
\text { Chá de }\end{array}$ & $\begin{array}{l}43 \\
52\end{array}$ & $\begin{array}{l}11,5 \\
14,0\end{array}$ & $\begin{array}{l}18 \\
28\end{array}$ & $\begin{array}{l}6,0 \\
9,2\end{array}$ & $\begin{array}{l}61 \\
80\end{array}$ & $\begin{array}{r}9,0 \\
11,9\end{array}$ \\
\hline $\begin{array}{l}\text { quina/quinino } \\
\text { Outras plantas } \\
\text { Outras }\end{array}$ & $\begin{array}{l}21 \\
51\end{array}$ & $\begin{array}{r}5,7 \\
13,7\end{array}$ & $\begin{array}{l}12 \\
34\end{array}$ & $\begin{array}{r}4,0 \\
11,2\end{array}$ & $\begin{array}{l}33 \\
85\end{array}$ & $\begin{array}{r}4,9 \\
12,5\end{array}$ \\
\hline $\begin{array}{l}\text { respostas } \\
\text { Não sabe/ }\end{array}$ & 12 & 3,2 & 12 & 4,0 & 24 & 3,6 \\
\hline $\begin{array}{l}\text { sem resposta } \\
\text { Não utiliza }\end{array}$ & $\begin{array}{r}1 \\
193\end{array}$ & $\begin{array}{r}0,2 \\
51,7\end{array}$ & $\overline{199}$ & $\overline{65,6}$ & $\begin{array}{r}1 \\
392\end{array}$ & $\begin{array}{r}0,1 \\
58,0\end{array}$ \\
\hline Total & 373 & 100,0 & 303 & 100,0 & 676 & 100,0 \\
\hline
\end{tabular}

Obs.: Foram consideradas as respostas múltiplas distribuídas nas categorias, com o objetivo de registrar todas as respostas obtidas.

riparoba, cidreira, flor de mamão macho, flor de batatão, folha de abacate, arnica, emburana, entre outras.

As freqüências maiores encontradas na utilização do alho e do boldo são justificadas pela cultura popular de que o cheiro do alho supostamente espanta mosquito e chás amargos como losna, boldo e quina sejam bons para o fígado, órgão atingido pela malária ${ }^{10}$.

Com relação ao tempo transcorrido entre os primeiros sintomas e a procura de diagnóstico na SUCEN (Tabela 5) nota-se que $68,2 \%$ dos indivíduos pesquisados procuraram o Labotarório de Malária tão logo apareceram qualquer suspeita ou sinais da

\section{TABELA 5}

Distribuição dos indivíduos segundo o período transcorrido entre os primeiros sintomas e a procura de diagnóstico na SUCEN - Laboratório de Malária da Região Metropolitana de São Paulo, novembro de 1986 a junho de 1987

\begin{tabular}{lrr}
\hline $\begin{array}{l}\text { Tempo transcorrido entre os primeiros } \\
\text { sintomas e a procura de diagnóstico }\end{array}$ & Ne & $\%$ \\
\hline Até 3 dias & 386 & 68,2 \\
De 4 a 6 dias & 98 & 17,3 \\
7 ou mais dias & 73 & 12,9 \\
Não sabe/sem respostas & 9 & 1,6 \\
\hline Total & 566 & 100,0 \\
\hline
\end{tabular}

doença. Esta procura precoce indica conhecimento do atendimento para malária oferecido pela SUCEN, além da noção dos sintomas característicos da doença ${ }^{16}$.

Quando pesquisado sobre a necessidade de procurar atendimento para malária após o aparecimento dos primeiros sintomas, dos 566 indivíduos, $541(96 \%)$ a consideram necessária, para a cura imediata da doença.

\section{CONCLUSÃO}

Considerando-se as ressalvas já referidas no texto no tocante à análise de algumas variáveis, pode-se concluir que a população de risco para a re-introdução da malária no Estado de São Paulo se constitui na grande maioria de individuos do sexo masculino e economicamente ativa, conhecedora do risco de contrair a doença em área endêmica. Esta população associa a malária à presença do vetor e tem consciência da necessidade de procurar atendimento médico tão logo se iniciem os primeiros sintomas, aspecto este de suma importância para a vigilância epidemiológica da doença no Estado de São Paulo. 
ALVES, M. J. C. P. et al. [Social and economic aspects of patients with imported malaria in the metropolitan region of S. Paulo City, S. Paulo State, Brazil. I - Characterization of the population and knowledge of the disease]. Rev. Saúde públ., S. Paulo, 24: 253-8, 1990.

ABSTRACT: With a view to discovering the social and economic characteristics of people from endemic malarial areas of Brazil, 566 suspected malaria cases were studied at the S. Paulo City Metropolitan Region Malaria Laboratory. Data were obtained by means of the application of standardized questionnaires over the period from November 1986 to June 1987 to individuals with as history of transit to Brazil's endemic malarial region. Of the population studied, $345(61.0 \%)$ lived in the endemic area; 479 $(84.6 \%)$ were males, $513(90.7 \%)$ were between 15 and 55 years old and $307(54.2 \%)$ presented positive plasmodium haemoscopia. The analysis by educational level indicated that $486(85.9 \%)$ had primary or secondary education; $109(19.3 \%)$ worked in mineral extraction; $74(13.2 \%)$ were farmers, and $46(8.1 \%)$ were related to terrestrial transportation activities. With respect, to knowledge of the disease, 384 $(67.8 \%)$ had at least 1 bout of malaria before and $491(86.8 \%)$ associated the disease with the presence of the vector. Of the 221 residents in S. Paulo, $207(93.7 \%)$, as well as $336(97.4 \%)$ of those residing in the endemic area already know of the riscks of infection prior to travelling through the area of transmission. The interval between the first symptoms and the seeking for medical care varied from 0 to 3 days in 386 cases $(68.2 \%)$. The frequencies of the variables studied analysed according to the haemoscopic result and the place of residence, were statistically significant.

KEYWORDS: Malaria, epidemiology. Socioeconomic factors. Knowledge, attitudes, practice.

\section{REFERÊNCIAS BIBLIOGRÁFICAS}

1. AGUDELO, S. F. Os processos sócio-econômicos na transmissão e no controle de malária. Rev. bras. Malar., 35: 89-100, 1983.

2. BARROS, M. B. de A. Saúde e classe social: um estudo sobre morbidade e consumo de medicamentos. $\mathrm{Ri}$ beirão Preto, 1983. [Tese de Doutoramento - Faculdade de Medicina de Ribeirão Preto da USP].

3. CARVAlHeiro, J. R. Processo migratório e disseminação de doenças. [Apresentado ao "Working Group on Geography of Health", Brasilia, 1982].

4. CARVALHO, F. M. et al. Morbidade referida e utilização de consulta médica em cinco populações do Estado da Bahia. Cienc. e Cult., 40: 853-8, 1988.

5. CONJUNTURA DEMOGRÁFICA. (Fundação SEADE). São Paulo, (1) out./dez. 1987.

6. IVERSSON, L. B. Significado do estudo de caso de epidemiologia. Cad. Saúde públ., Rio de Janeiro, 3: 16-21, 1987.

7. MARQUES, A. C. Migrações intemas e dispersão de endemias. Saúde Brasil, 1: 143-51, 1983.

8. MARQUES, A. C. Situação da malária no Brasil. Hiléia med., 8: 95-6, 1987.

9. MINTSTÉRIO DA SAÚDE. Superintendência de Campanhas de saúde pública. Relatório anual. Brasília, 1987.

10. PENNA, M. Dicionário brasileiro de plantas medicinais.
Descrição das plantas medicinais indigenas e das exóticas aclimadas no Brasil. $3^{\text {a }}$ ed. Rio de Janeiro, Kosmos, 1946.

11. SAWYER, D. R. et al. Malaria on Amazon frontier: economic and social aspects of transmission and control. Belo Horizonte, CEDEPLAR/UFMG, 1987. [Relatório de Pesquisa].

12. SECRETARIA DE ESTADO DA SAÚDE. Superintendência de Controle de Endemias (SUCEN). Relatório da avaliação epidemiológica do Programa de Controle da Malária no Estado de São Paulo. São Paulo, 1987. [Mimeografado].

13. TAUIL, P. L. Malária: agrava-se o quadro da doença no Brasil. Ciênc. Hoje, 2(12): 58-64, 1984.

14. WANDERLEY, D. M. V. et al. Malária no Estwado de São Paulo, Brasil, 1980 a 1983. Rev. Saúde públ., S. Paulo, 19: 28-36, 1985.

15. WANDERLEY, D. M. V. et al. Contribuição para as informações epidemiológicas de malária no Estado de São Paulo, Brasil. Rev. Soc. bras. Med. trop., 20: 143-6, 1987.

16. WANDERLEY, D. M. V. et al. Malária no Estado de São Paulo: avaliação de aspectos da vigilância epidemiológica. Cad. Saúde públ. Rio de Janeiro, 5: 296.304, 1989.

Recebido para publicação em 12/7/1989 Reapresentado em 21/3/1990 Aprovado para publicação em 9/4/1990 